\title{
REARRANJO DOS FATORES DE PRODUÇÃO NO PROCESSO PRODUTIVO DE CEREAIS: A IMPORTÂNCIA DO FATOR TECNOLOGIA
}

\footnotetext{
Felipe Dalzotto Artuzo ${ }^{1}$, Cristian Rogério Foguesatto ${ }^{2}$, Antônio Luiz Fantinel ${ }^{3}$

${ }^{1}$ Engenheiro Agrônomo, Doutorando do Programa de Pós-Graduação em Agronegócios - UFRGS, Porto Alegre, RS, Brasil. E- mail: felipeartuzo1@hotmail.com

${ }^{2}$ Bacharel em Administração, Doutorando do Programa de Pós-Graduação em Agronegócios - UFRGS, Porto Alegre, RS, Brasil.

${ }^{3}$ Tecnólogo em Agronegócio, Doutorando do Programa de Pós-Graduação em Agronegócios - UFRGS, Porto Alegre, RS, Brasil.

Recebido em: 03/10/2016 - Aprovado em: 21/11/2016 - Publicado em: 05/12/2016 DOI: 10.18677/EnciBio_2016B_086
}

\begin{abstract}
RESUMO
A mudança na alocação dos fatores de produção, no processo produtivo, desempenhará um papel decisivo para atender às demandas futuras de alimentos. Nesse sentido, o artigo tem por objetivo analisar o rearranjo dos fatores de produção a partir da inclusão da tecnologia no processo de produção agrícola. Os países analisados foram o Brasil, o EUA e a China. Os dados de produção, produtividade e área foram obtidos pela Organização das Nações Unidas para Alimentação e Agricultura $(\mathrm{FAO})$. As informações referentes à população foram obtidas pelo World Bank. Adotou-se como ferramentas de análises a variação (\%), índices de IEPP e $I_{E P A}$, correlação e regressão. A variação positiva, quanto à produção e produtividade, foi percebida nos três países. Sendo que os EUA reduziu sua área de cultivo de cereais em $10,60 \%$. A redução da área, com o aumento da produção, refere-se, em partes, ao fator tecnologia alocado no processo de produção. No Brasil, o fator trabalho é substituído pelo fator tecnologia. Sendo que a população rural decresce, em valores relativos e absolutos, enquanto a produtividade aumenta. Por outro lado, a China, por questões demográficas, alia tecnologia, trabalho e terra, no seu sistema produtivo, oscilando-os durante o período de análise.
\end{abstract}

PALAVRAS-CHAVE: Agronegócios; Mudança tecnológica; Economia rural.

\section{REARRANGEMENT OF THE PRODUCTION FACTORS IN THE PRODUCTION PROCESS OF CEREAL: IMPORTANCE OF TECHNOLOGY FACTOR}

\begin{abstract}
Change in the allocation of factors of production in production process will result in a important role to supply future demands of food. In this context, the article aims to analyze the rearrangement of production factors from the inclusion of technology in the agricultural production process. The countries surveyed were Brazil, USA and China. The data of production, and area were obtained by the Food and Agriculture Organization (FAO). Data about population were by the World Bank.It was adopted as the variation analysis tools (\%), IEPP and IEPA indices, correlation and regression. The positive variation in production and productivity was perceived in the three ENCICLOPÉDIA BIOSFERA, Centro Científico Conhecer - Goiânia, v.13 n.24; p.905 2016
\end{abstract}


countries. The USA reduced the cereal area in $10.60 \%$. This reduction, in parallel to increase production refers, in part, with technology factor allocated in the production process. In Brazil, labor factor is substituted by the technology factor. In this case, the rural population decreased in absolute and relative values, while productivity increases. On the other hand, China, due to their demographic characteristics combines technology, labor and land in the productive system, ranging them during this analysis.

KEYWORDS: Agribusiness; Technological change; Rural economy.

\section{INTRODUÇÃO}

O agricultor que exerce a atividade agrícola de maneira idêntica a de seus antepassados não tem potencial de produzir alimentos em quantidade suficiente para a demanda crescente, apesar da riqueza da terra ou da intensidade do seu trabalho (SCHULTZ, 1964). Nesse contexto, o autor frisa a importância das inovações e a modernização gradual da agricultura. Antes de Schultz, Thomas Malthus, em seu ensaio pessimista em relação à população, afirmou que o crescimento populacional seria restringido por uma taxa de crescimento lento na produção de alimentos (MALTHUS, 1872). Uma das principais deficiências do seu ensaio foi à subestimação da mudança tecnológica na agricultura (TREWAVAS, 2002), o que SCHULTZ (1964) apontou como base fundamental no processo de produção de alimentos.

No entanto, na época de Malthus, a mudança tecnológica era insignificante. A produção de alimentos era baseada, exclusivamente, no aumento dos fatores de produção, como trabalho e terra. Porém, a partir do século XIX, as inovações tornaram-se fundamentais, mudando a lógica dos fatores na produção de alimentos (RUNGE et al., 2003; FEDERICO, 2005). Desde então, o uso eficiente da terra, a partir da incorporação de tecnologias, tem sido um dos principais pontos do crescimento da produção agrícola (BROWN \& WALDRON, 2013; WANG \& ZHOU, 2013).

A importância da tecnologia, para o setor agrícola, é amplamente conhecida na literatura científica (DOW \& REED, 2011; BRITE \& MARSTON, 2013; LONG et al., 2016). Alguns estudos documentam a necessidade da inovação para satisfazer a demanda global de alimentos, além de mantê-los a preços toleráveis (ALSTON et al., 2009; LÄPPLE et al., 2015; LÄPPLE et al., 2016). O aumento da produção tem possibilitado o crescimento demográfico e econômico de muitas regiões. Por exemplo, o crescimento no setor agrícola tem um impacto maior sobre a redução da pobreza na África e na Ásia do que em outros setores (THIRTLE et al., 2003).

Não há razões que impeçam o setor agrícola, de qualquer país, de contribuir consideravelmente para o crescimento econômico. Naturalmente, uma agricultura baseada no uso inadequado dos fatores de produção, não pode gerar bons resultados. Entretanto, uma agricultura modernizada, readequando os fatores de produção, agregando tecnologia, é capaz de gerar ótimos resultados.

Nesse sentido, com uma população mundial em expansão, juntamente com a preocupação com a escassez de recursos, com a disponibilidade de terras, a conservação da biodiversidade e o aquecimento global (FOLEY et al., 2011), o estíulo à adoção e difusão de tecnologias agrícolas torna-se fundamental. Dessa forma, o estudo tem por objetivo analisar o rearranjo dos fatores de produção a partir da inclusão da tecnologia no processo de produção agrícola. 


\section{MATERIAL E METODOS}

O artigo está construído sob o enfoque da teoria da produção, subdivisão da teoria neoclássica da firma, estando presente em texto de PINDYCK \& RUBINFELD (2002). O estudo foi realizado a partir do levantamento de dados da Organização das Nações Unidas para Alimentação e Agricultura (FAO) e do World Bank.

A pesquisa se fundamenta nos dados históricos da área agrícola arável, produção e produtividade de cereais, determinados e ponderados conforme a metodologia da FAO (2016). Os valores totais dos cereais integram as culturas do trigo, arroz, cevada, milho, centeio, aveia, sorgo, triticale e trigo sarraceno. Da mesma forma, foram utilizados os dados populacionais (população total, população urbana e rural), conforme disponibilizados pelo WORLD BANK (2016). Os parâmetros adotados foram: (a) área, (b) produção, (c) produtividade, (d) população total, (e) população urbana e (f) população rural. O período de análise compreende os anos de 1961 a 2014.

O estudo foi realizado em três países: (a) Brasil, (b) EUA e (c) China. Sendo possível avaliar os fatores (a) trabalho, (b) tecnologia e (c) terra. Levando em consideração as características de cada país, bem como o fator predominante do processo produtivo agrícola.

\section{Descrição da análise}

Análise da variação (\%)

Com a finalidade de determinar a variação da área, da produção, da produtividade e da população, utilizou-se à equação da variação percentual (1) que é adotada para descrever a relação entre um valor ou quantidade anterior e um valor ou quantidade posterior.

Variação $(\%)=\left(\frac{v_{2}-v_{1}}{v_{1}}\right) \times 100$

Em que:

$v_{1}=$ Valor anterior;

$v_{2}=$ Valor posterior;

A análise foi realizada para determinar qual parâmetro, de cada país, sofreu variação ao longo do período. Servindo como auxílio para interpretação no rearranjo dos fatores de produção (tecnologia, trabalho e terra).

Análise de correlação

Para determinar a força de associação entre o parâmetro "população rural", com a "área, produção e produtividade", adotou-se a correlação de Pearson (2), tendo em vista a normalidade dos dados. A população rural correlacionada foi à variação anual, levando em consideração o ano base de 1961.

$r=\frac{\sum_{i}\left(x_{i}-\bar{z}\right)\left(y_{i}-\bar{z}\right)}{\sqrt{\sum_{i}\left(x_{i}-\bar{x}\right)^{2} \sum_{i}\left(y_{i}-\bar{y}\right)^{2}}}$

Em que:

$x_{1}, x_{2}, x_{3}, \ldots, x_{n}$ e $y_{1}, y_{2}, y_{3}, \ldots, y_{n}=$ são os valores medidos para ambas as variáveis. 
O objetivo da aplicação da análise de correlação foi entender 0 comportamento entre os parâmetros estudados. Desta forma, sendo possível atribuir inferências entre o aumento e/ou redução da população rural com os parâmetros de área, produção e produtividade dos países analisados.

Índices de $I_{E P P}$ e I

Os índices de eficiência produtiva e de área, em relação à população rural, são medidos pelo $I_{E P P}$ e $I_{E P A}$, respectivamente. Os índices levam em consideração a produção pela população rural (3) e a área pela população rural (4), durante o período analisado.

$$
I_{E P P}=\frac{P_{Y}}{p}
$$

Em que:

$P_{r}=$ Valor total da produção (em toneladas) de cereais do ano analisado;

$P=$ Valor total da população rural do ano analisado

$l_{E P A}=\frac{A}{P}$

Em que:

$A=$ Valor total da área (em hectares) destinada ao cultivo de cereais, no ano analisado;

$P=$ Valor total da população rural do ano analisado.

Análise de regressão

A análise de regressão (5) foi utilizada para explorar e inferir a relação de uma variável dependente para outra variável independente específica. Neste sentido, adotou-se como variável dependente a população rural e como variável independente a produtividade.

$f=\beta_{0}+\beta_{1} x+\beta_{2} x^{2}+\cdots+\beta_{m} x^{m}+e$

Em que:

$f$ = variável aleatória e representa o valor da variável resposta na i-ésima observação;

$x_{i}=$ representa o valor da variável explicativa na i-ésima observação;

$\beta_{0}, \beta_{1}, \beta_{2}, \ldots, \beta_{m}=$ parâmetros do modelo, que serão estimados, e que definem a reta de regressão;

$e=$ erro experimental

\section{RESULTADO E DISCUSSÃO}

A demanda por culturas agrícolas, para a alimentação humana, ração animal e combustível, tem aumentado nos últimos anos (ZHOU \& STAATZ, 2016; DAVIS et al., 2016). No entanto, os recentes aumentos no consumo de carnes, nas economias emergentes, juntamente com a aceleração no uso de grãos para a produção de biocombustíveis, têm pressionado os estoques mundiais. Para satisfazer a crescente demanda mundial, duas opções estão disponíveis: (1) o aumento da área para ENCICLOPÉDIA BIOSFERA, Centro Científico Conhecer - Goiânia, v.13 n.24; p.908 2016 
produção agrícola ou (2) o aumento da produtividade com a quantidade de terra já existente (LAMBIN \& MEYFROIDT, 2011).

Nesse sentido, analisando o histórico da produção e produtividade dos principais cereais definidos pela FAO (2016), aliado a área agrícola destinada para sua produção, o aumento da produção agrícola foi obtido pelo rearranjo dos fatores de produção. Os três países (Brasil, EUA e China) analisados obtiveram aumento na produção no decorrer das últimas décadas. O Brasil apresentou a maior variação, sendo essa de 574,35\%, seguido pela China e EUA, com 410,04\% e 170,71\%, respectivamente. Em termos de valores absolutos de produção, a China ocupa o primeiro lugar, com 559,313 milhões de toneladas, seguido por EUA e Brasil, com respectivos 442,933 e 101,398 milhões de toneladas (Tabela 1).

TABELA 1 - Variação da produção, produtividade e da área cultiva de cereais, no Brasil, EUA e China, no período de 1961 a 2014.

\begin{tabular}{|c|c|c|c|c|c|c|}
\hline & \multicolumn{2}{|c|}{ Brasil } & \multicolumn{2}{|c|}{ EUA } & \multicolumn{2}{|c|}{ China } \\
\hline & Produção $^{a}$ & Variação $^{b}$ & Produção & Variação & Produção & Variação \\
\hline 1961 & 15,036 & - & 163,620 & - & 109,660 & - \\
\hline 1970 & 23,698 & 57,61 & 186,861 & 14,20 & 200,837 & 83,15 \\
\hline 1980 & 33,217 & 120,91 & 269,884 & 64,95 & 280,287 & 155,60 \\
\hline 1990 & 32,490 & 116,08 & 312,411 & 90,94 & 404,719 & 269,07 \\
\hline 2000 & 45,893 & 205,22 & 342,628 & 109,40 & 407,337 & 271,45 \\
\hline 2010 & 75,161 & 399,86 & 401,670 & 145,49 & 498,468 & 354,56 \\
\hline \multirow[t]{2}{*}{2014} & 101,398 & 574,35 & 442,933 & 170,71 & 559,313 & 410,04 \\
\hline & Produt. $^{c}$ & Variação & Produt. & Variação & Produt. & Variação \\
\hline 1961 & 1346,3 & - & 2522,3 & - & 1192,7 & - \\
\hline 1970 & 1408,8 & 4,64 & 3154,9 & 25,08 & 2127,7 & 78,39 \\
\hline 1980 & 1575,7 & 17,04 & 3771,9 & 49,54 & 2937,3 & 146,27 \\
\hline 1990 & 1755,1 & 30,36 & 4755,1 & 88,52 & 4320,9 & 262,28 \\
\hline 2000 & 2661,4 & 97,68 & 5854,3 & 132,10 & 4752,6 & 298,47 \\
\hline 2010 & 4040,8 & 200,14 & 6987,6 & 177,03 & 5526,7 & 363,38 \\
\hline \multirow[t]{2}{*}{2014} & 4640,5 & 244,69 & 7637,4 & 202,80 & 5886,4 & 393,54 \\
\hline & Área $^{\mathrm{d}}$ & Variação & Área & Variação & Área & Variação \\
\hline 1961 & 11,169 & - & 64,870 & - & 90,553 & - \\
\hline 1970 & 16,822 & 50,62 & 59,229 & $(8,69)$ & 93,712 & 3,49 \\
\hline 1980 & 21,081 & 88,75 & 71,550 & 10,30 & 95,054 & 4,97 \\
\hline 1990 & 18,512 & 65,75 & 65,700 & 1,28 & 93,583 & 3,35 \\
\hline 2000 & 17,244 & 54,40 & 58,526 & $(9,78)$ & 85,640 & $(5,43)$ \\
\hline 2010 & 18,600 & 66,54 & 57,483 & $(11,39)$ & 90,173 & $(0,42)$ \\
\hline 2014 & 21,851 & 95,64 & 57,996 & $(10,60)$ & 94,997 & 4,91 \\
\hline
\end{tabular}

Nota: ${ }^{a}$ produção em mil toneladas; ${ }^{0}$ variação em \%; ${ }^{c}$ produtividade em $\mathrm{kg} \mathrm{ha}{ }^{-1} ;{ }^{\mathrm{d}}$ área em $10^{6} \mathrm{ha}$.

Fonte: Elaborado a partir dos dados da FAO (2016).

O crescimento dos valores absolutos de produção está aliado, em partes, ao aumento do fator terra. Brasil e China obtiveram incremento de área de $95,64 \%$ e 4,91\%, em 2014 com relação ao período de 1961. Em contrapartida, os EUA reduziu a área cultivada de cereais $(-10,60 \%)$. Mesmo assim, houve incremento da produção, ocasionado pelo aumento da produtividade. Esse aumento, aliado com a redução da área, pode ser explicado pelo fator tecnologia. Países como o Brasil e a 
China, mesmo não havendo redução da área, obtiveram aumento da produção, ocasionado, em partes, pela mudança tecnológica.

Nos países em desenvolvimento, existem agricultores com alto e baixo nível de desenvolvimento tecnológico. O Brasil é exemplo disso (VIEIRA FILHO \& SILVEIRA, 2012). A agricultura brasileira apresenta agricultores que empregam modernas técnicas produtivas, recomendadas pelos centros de experimentação e pesquisa. Mas também, há agricultores em estágio atrasado de desenvolvimento, que mantêm baixos níveis de adoção e conhecimento tecnológico. Assim, existem processos de produção que predominam tecnologia, ao invés de trabalho, e outro com predominância de trabalho, ao invés de tecnologia. Alguns, até, aliando ambos.

Os EUA, país desenvolvido, apresenta alto nível tecnológico aplicado na agricultura (VIEIRA FILHO \& SILVEIRA, 2012), preconizando, desta forma, o fator tecnologia. Em compensação a China, país com economia pujante, enfoca e dá ênfase ao fator trabalho, fato esse explicado pela alta densidade demográfica. Nesse sentido, a variável população torna-se fundamental para explicar a mudança no rearranjo dos fatores de produção. Ressalta-se que, mesmo focando o fator trabalho, há adoção de tecnologias, mas em menor proporção quando comparados com outros países (ZHANG et al. 2016).

Nos últimos anos, ocorreram mudanças na configuração demográfica, entre o meio urbano e rural (FAO, 2016). Em 1960, Brasil, EUA e China, apresentavam uma população rural de 39,05; 54,21 e 558,98 milhões de habitantes, respectivamente. Já, em 2014, essa população passou a ser de 30,02; 59,16 e 621,97 milhões de habitantes (Tabela 2). Apenas no Brasil, em valores absolutos, houve redução da população rural. Mas, levando em consideração valores relativos entre a população total, os três países apresentaram redução na porcentagem da população rural. Destacando o Brasil com uma taxa de $86 \%$ de população urbana.

TABELA 2 - Variação da população total, urbana e rural, no Brasil, EUA e China, no período de 1961 a 2014.

\begin{tabular}{|c|c|c|c|c|}
\hline & País & 1960 & 2014 & Variação (\%) \\
\hline \multirow[b]{3}{*}{ População Total (milhões) } & Brasil & 72,49 & 206,08 & 184,27 \\
\hline & EUA & 180,67 & 318,86 & 76,48 \\
\hline & China & 667,07 & 1364,27 & 104,52 \\
\hline \multirow[b]{3}{*}{ População Urbana (milhões) } & Brasil & 33,45 & 176,06 & 426,37 \\
\hline & EUA & 126,46 & 259,70 & 105,36 \\
\hline & China & 108,09 & 742,30 & 586,77 \\
\hline \multirow[b]{3}{*}{ População rural (milhões) } & Brasil & 39,05 & 30,02 & $-23,12$ \\
\hline & EUA & 54,21 & 59,16 & 9,13 \\
\hline & China & 558,98 & 621,97 & 11,27 \\
\hline \multicolumn{5}{|c|}{ População rural e urbana em relação à população total } \\
\hline \multirow{6}{*}{ População Urbana (\%) } & Brasil & 46,14 & 85,43 & \\
\hline & EUA & 70,00 & 81,45 & \\
\hline & China & 16,20 & 54,41 & \\
\hline & Brasil & 53,86 & 14,57 & \\
\hline & EUA & 30,00 & 18,55 & \\
\hline & China & 83,80 & 45,59 & \\
\hline
\end{tabular}

Fonte: Elaborado a partir dos dados do WORLD BANK (2016). 
O índice da população rural, a partir de 1961, está correlacionado com a produção, área e produtividade. Ao passo em que se muda a configuração demográfica, em que a maior parte da população torna-se urbana, aumenta-se a produção e produtividade dos cereais. Fato esse demonstrado pela forte força de associação negativa entre a população rural com a produção (Brasil: -0,875; EUA: 0,880 e China: $-0,886$ ) e produtividade (Brasil: -0,853; EUA: $-0,961$ e China: $-0,906$ ) (Tabela 3). Da mesma forma, mas com menor força de associação, a redução do índice da população rural tende a estar correlacionada com a área (Brasil: -0,592; EUA: $-0,494$ e China: -0,499). A explicação para este fato pode estar no aumento da área agrícola arável.

TABELA 3 - Matriz correlação entre os parâmetros "produção", "área", "produtividade" e "população rural", no Brasil, EUA e China, no período de 1961 a 2014.

\begin{tabular}{|c|c|c|c|c|}
\hline & Produção & Área & Produtividade & População Rural \\
\hline \multicolumn{5}{|c|}{ Brasil } \\
\hline $\begin{array}{l}\text { Produção } \\
\text { Área }\end{array}$ & $\begin{array}{c}1 \\
503^{* *}\end{array}$ & 1 & & \\
\hline Produtividade & $977^{* *}$ &, $325^{*}$ & 1 & \\
\hline População Rural &,$- 875^{* *}$ &,- 592 &,$- 853^{* *}$ & 1 \\
\hline \multicolumn{5}{|c|}{ EUA } \\
\hline Produção & 1 & & & \\
\hline Área &,- 118 & 1 & & \\
\hline Produtividade & , $952^{* \star}$ &,$- 411^{\star *}$ & 1 & \\
\hline População Rural &,$- 880^{* *}$ &, $494^{* *}$ &,$- 961^{* *}$ & 1 \\
\hline \multicolumn{5}{|c|}{ China } \\
\hline Produção & 1 & & & \\
\hline Área & -349 & 1 & & \\
\hline Produtividade & ,988* &,$- 483^{* *}$ & 1 & \\
\hline População Rural &,- 886 & (499* &,$- 906^{\star \star}$ & 1 \\
\hline
\end{tabular}

${ }^{*}$ Correlação de Pearson significante a nível de $p<0,01$

Fonte: Elaborado a partir dos dados do WORLD BANK (2016) e FAO (2016)

Quando analisado os índices de $\mathrm{I}_{\text {EPP }}$ e $\mathrm{I}_{\text {EPA }}$, é possível inferir o efeito dos fatores tecnologia, trabalho e terra. O I IPP dos EUA despontou em relação ao do Brasil e da China (Figura 1a). Assim sendo, a eficiência no processo produtivo dos EUA é superior ao dos outros países de estudo. A produção, em tonelada, de cada produtor rural americano é superior à produção de um produtor brasileiro e chinês. Sendo que a população rural, em valor relativo, reduziu para todos os países. Assim, o aumento da produção dos EUA se deve, em partes, pelo fator tecnologia. 

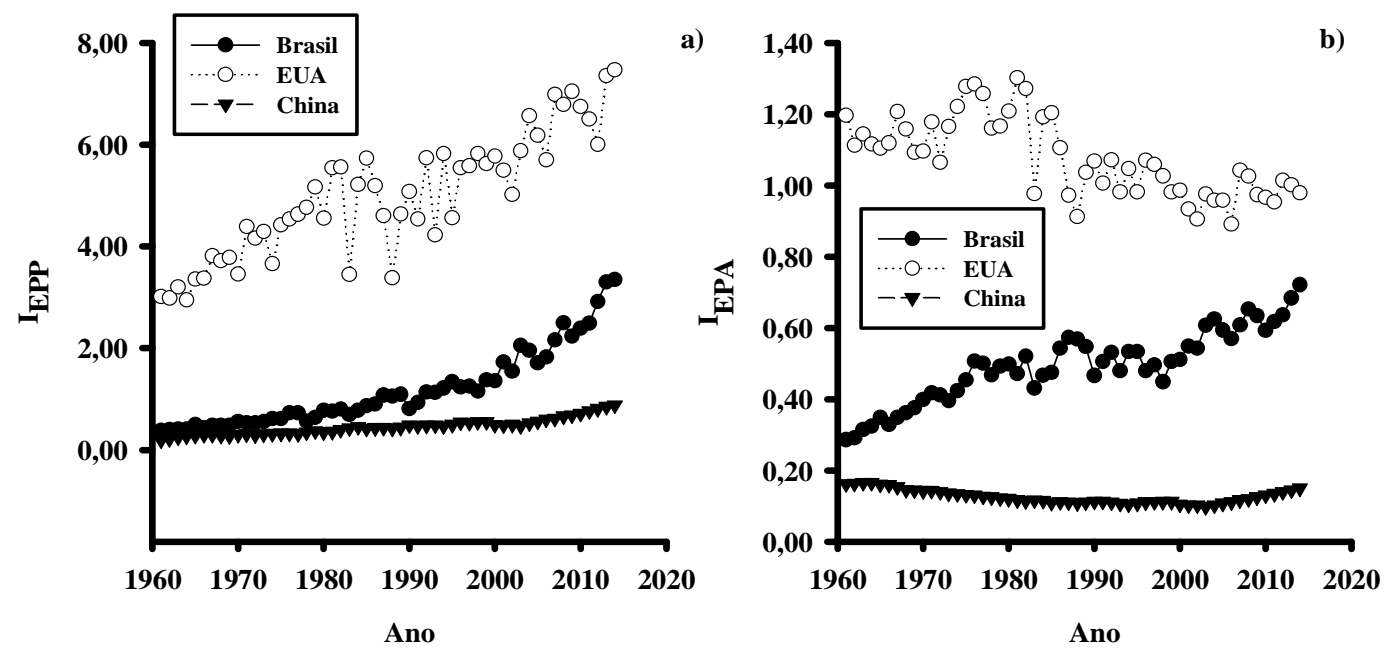

FIGURA 1- Índice de IEPP (a) e I IEPA (b) no Brasil, EUA e China, no período de 1961 a 2014.

Fonte: Elaborado a partir dos dados do WORLD BANK (2016) e FAO (2016)

Em relação ao $I_{E P A}$, os EUA, novamente, apresenta o maior índice. Sendo que a maior quantidade de área, por produtor rural, destinado à produção de cereais, é dos americanos (Figura 1b). $O$ índice americano manteve constante nos últimos anos, com leves oscilações. Enquanto isso, o índice brasileiro tem aumentado. Com a redução da população rural, tanto em valores relativos como absolutos, a área agrícola arável do Brasil passou a ser cultivada por um número menor de produtores. Com isso, aliado ao aumento da área por produtor, facilitou-se a adoção e difusão de tecnologias agrícolas.

O fator tecnologia é um dos principais responsável pelo aumento da produção, por meio do aumento da produtividade. O aumento da área, por produtor rural, favoreceu a mudança tecnológica, sendo possível diluir os custos por uma quantidade maior de área. Alguns países, como o Brasil, seguem uma tendência. Ao passo que há uma redução da população rural, há, também, um aumento da produtividade. A equação da Figura 2 a possibilita explicar em $92,06 \%$ dos casos observados, a relação da população rural com produtividade. Já para os EUA e China, a equação explica $66,37 \%$ (Figura 2b) e 93,83\% (Figura 2c) dos casos, respectivamente.

Dessa forma, retornando ao passado e pensando no futuro, é necessário frisar as ideias que SCHULTZ (1964) apontava em meados do século XX. Segundo o autor, um país dependente de uma agricultura tradicional, baseada apenas no trabalho e terra, é um país pobre. E, por ser pobre, gasta a maior parte de sua renda em alimentos. Mas quando um país desenvolve o seu setor agrícola, os alimentos tornam-se abundantes, liberam-se braços do setor agrícola para o industrial, a renda cresce e uma parte menor dela é empregada na aquisição de alimentos. 

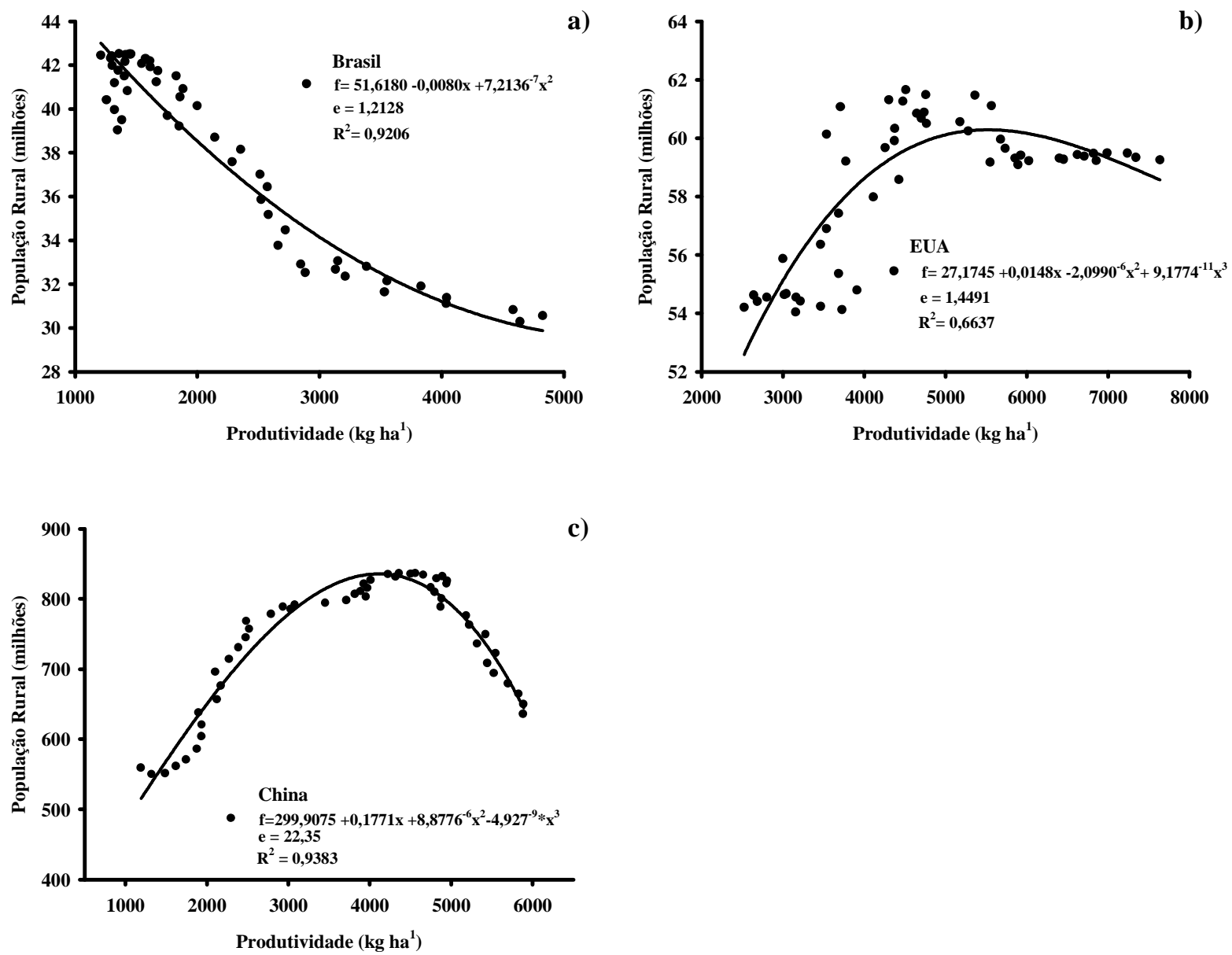

FIGURA 2 - Relação entre produtividade e população rural no Brasil (a), EUA (b) e China (c), no período de 1961 a 2014.

Fonte: Elaborado a partir dos dados do WORLD BANK (2016) e FAO (2016)

Exemplo disso pode ser a produção de grãos na China, no qual aumentou dramaticamente durante os últimos 20 anos (DENG et al, 2015a.; DENG et al, 2015b). Fato explicado, principalmente, pela intensificação de terras, com a adoção de novas tecnologias (novas variedades e cultivares) (CHEN et al., 2013). No futuro, a demanda por produtos agrícolas vai continuar a aumentar significativamente. No entanto, esse aumento da procura dificilmente será atendida pela expansão da área cultivada, devido à escassez de terras produtivas e à crescente demanda de utilizações de terras não agrícolas (JIANG et al., 2011). Assim, tornando-se necessário otimizar o fator tecnologia, a partir de adoção e difusão de inovações tecnológicas.

E fundamental o rearranjo dos fatores de produção, adotando e trabalhando a questão tecnológica em um sistema de produção. Ao passo que o aumento da produção agrícola não esteja atribuído ao aumento de áreas agrícolas, pois ocasionaria outras implicações, como, por exemplo, o desmatamento (RAMANKUTTY et al., 2008). Dessa forma, o aumento da eficiência por área, aumentando a produtividade dos países, implica na adoção de tecnologias agrícolas. Sendo essa proporcional à necessidade específica de cada caso e/ou unidade de produção. 


\section{CONCLUSÕES}

Em suma, o rearranjo dos fatores de produção torna-se necessário quando se pensa a longo prazo. A agricultura que ficar refém, apenas, dos fatores "terra" e "trabalho", não poderá produzir alimentos em quantidade suficiente. Nessa perspectiva, não importa o quão rica seja a terra e/ou o quanto de unidade de trabalho for alocada nela. O desenvolvimento tecnológico, a partir da tecnologia, passou a ser considerado, efetivamente, como um fator de produção, em função do considerável desempenho econômico obtido por países que pautaram seu desenvolvimento nesse fator, como, por exemplo, os EUA.

O conhecimento que torna possível a transformação é uma forma de "capital", que requer investimentos. Investimentos não apenas em insumos materiais, nos quais se encaixa uma parte do conhecimento gerado, mas também, em um grau mais além. O investimento no "pessoal do campo". Esses que aplicam a tecnologia na produção. Os quais optam por um processo de produção, entre uma gama de processos, que possibilite o maior retorno econômico para sua atividade.

A redução da população rural no Brasil, em valores absolutos e relativos, demonstra a tendência na substituição de "unidades de trabalho" por "unidades de tecnologia". Fato esse que não ocorre igualmente na China, por outras questões que estão além da necessidade da mudança. A população crescente da China cria a necessidade da predominância do fator "trabalho", tanto por questões demográficas, quanto pelo fator "terra" (área agricultável). Mesmo assim, a alta variação da produtividade pode estar atribuída, em partes, ao fator "tecnologia". Já os EUA, consegue aliar os fatores "terra", "trabalho" e "tecnologia". Sendo que reduziu a área para produção de cereais, em relação à área de 1961, mas aumentou a produção, a partir da tecnologia alocada em seu processo produtivo.

Por fim, o artigo não tem por pretensão criticar os processos produtivos dos países, mas apontar como os fatores estão sendo alocados. Nesse sentido, os três países analisados podem servir como exemplos de diferentes formas de implementação do fator tecnologia no processo produtivo global de cereais.

\section{REFERÊNCIAS}

ALSTON, J. M.; BEDDOW, J. M.; PARDEY, P.G. Agricultural research, productivity, and food prices in the long run. Science, v. 325, n. 5945, p. 1209-1210, 2009. Disponível em: <http://dx.doi.org/10.1126/science.1170451>. doi:10.1126/science. 1170451

BRITE, E. B.; MARSTON, J. M. Environmental change, agricultural innovation, and the spread of cotton agriculture in the Old World. Journal of Anthropological Archaeology, v. 32, n. 1, p. 39-53, 2013. Disponível em: <http://dx.doi.org/ 10.1016/j.jaa.2012.08.003 >. doi: 10.1016/j.jaa.2012.08.003

BROWN, C.; WALDRON, S. Agrarian change, agricultural modernization and the modelling of agricultural households in Tibet. Agricultural Systems, v. 115, p. 8394, 2013. Disponível em: <http://dx.doi.org/10.1016/j.agsy.2012.09.008 >. doi: 10.1016/j.agsy.2012.09.008

CHEN, Y; WU, Z. G.; ZHU, T. H.; Lei, Y. A. N. G.;CHIEN, H. P. Agricultural policy, climate factors and grain output: Evidence from household survey data in rural China. Journal of Integrative Agriculture, v. 12, n. 1, p. 169-183, 2013. Disponível 
em: <http://dx.doi.org/10.1016/S2095-3119(13)60217-8 >. doi: 10.1016/S2095$3119(13) 60217-8$

DAVIS, K. F; GEPHART, J. A.; EMERY, K. A.; LEACH, A. M.; GALLOWAY, J. N.; D'ODORICO, P. Meeting future food demand with current agricultural resources. Global Environmental Change, v. 39, p. 125-132, 2016. Disponível em: $<$ http://dx.doi.org/10.1016/j.gloenvcha.2016.05.004>.

doi:10.1016/j.gloenvcha.2016.05.004

DENG, X.; HUANG, J.; ROZELLE, S.; ZHANG, J.; LI, Z. Impact of urbanization on cultivated land changes in China. Land Use Policy, v. 45, p. 1-7, 2015a. Disponível em: $\quad$ http://dx.doi.org/10.1016/j.landusepol.2015.01.007 >. doi: 10.1016/j.landusepol.2015.01.007

DENG, X.; SHI, Q.; ZHANG, Q.; SHI, C.; YIN, F. Impacts of land use and land cover changes on surface energy and water balance in the Heihe River Basin of China, 2000-2010. Physics and Chemistry of the Earth, Parts A/B/C, v. 79, p. 2-10, 2015b. Disponível em: <http://dx.doi.org/10.1016/j.pce.2015.01.002>. doi: 10.1016/j.pce.2015.01.002

DOW, G. K.; REED, C. G. Stagnation and innovation before agriculture. Journal of Economic Behavior \& Organization, v. 77, n. 3, p. 339-350, 2011. Disponível em: $<$ http://dx.doi.org/10.1016/j.jebo.2010.11.006 >. doi: 10.1016/j.jebo.2010.11.006

FAO - FOOD AND AGRICULTURE ORGANIZATION. Production. 2016. Disponível em: < http://faostat3.fao.org/download/Q/*/E>. Acesso em: 05/06/2016.

FEDERICO, G. Feeding the world: an economic history of agriculture, 18002000. Princeton University Press, 2005.

FOLEY, J. A. et al. Solutions for a cultivated planet. Nature, v. 478, n. 7369, p. 337342, 2011. Disponível em: <http://dx.doi.org/10.1038/nature10452 >. doi: 10.1038 /nature10452

JIANG, Q.; DENG, X.; ZHAN, J.; HE, S. Estimation of land production and its response to cultivated land conversion in North China Plain. Chinese Geographical Science, v. 21, n. 6, p. 685-694, 2011. Disponível em: <http://dx.doi.org/10.1007/s11769-011-0479-8 >. doi: 10.1007/s11769-011-0479-8

LAMBIN, E. F.; MEYFROIDT, P. Global land use change, economic globalization, and the looming land scarcity. Proceedings of the National Academy of Sciences, v. 108, n. 9, p. 3465-3472, 2011. Disponível em: <http://dx.doi.org/ 10.1073/pnas. 1100480108 >. doi: 10.1073/pnas. 1100480108

LÄPPLE, D.; RENWICK, A.; THORNE, F. Measuring and understanding the drivers of agricultural innovation: Evidence from Ireland. Food Policy, v. 51, p. 1-8, 2015. Disponível em: <http://dx.doi.org/10.1016/.foodpol.2014.11.003 >. doi: 10.1016/j.foodpol.2014.11.003 
LÄPPLE, D.; RENWICK, A.; CULLINAN, J.; THORNE, F. What drives innovation in the agricultural sector? A spatial analysis of knowledge spillovers. Land Use Policy, v. 56,2016 p. 238-250, Disponível em: <http://dx.doi.org/10.1016/j.landusepol.2016.04.032 >. doi: 10.1016/j.landusepol.2016.04.032

LONG, T. B.; BLOK, V.; CONINX, I. Barriers to the adoption and diffusion of technological innovations for climate-smart agriculture in Europe: evidence from the Netherlands, France, Switzerland and Italy. Journal of Cleaner Production, v. 112, p. 9-21, 2016. Disponível em: <http://dx.doi.org/10.1016/j.jclepro.2015.06.044 >. doi: 10.1016/j.jclepro.2015.06.044

MALTHUS, T. R. An Essay on the Principle of Population Or a View of Its Pastand Present Effects on Human Happiness, an Inquiry Into Our Prospects Respecting the Future Removal Or Mitigation of the Evil swhich it Occasions by. Reeves and Turner, 1872.

RAMANKUTTY, N; EVAN, A. T.; MONFREDA, C.; FOLEY, J. A. Farming the planet: 1. Geographic distribution of global agricultural lands in the year 2000. Global Biogeochemical Cycles, v. 22, n. 1, 2008. Disponível em: <http://dx.doi.org/10.1029/2007GB002952>. doi: 10.1029/2007GB002952

RUNGE, C. F.; SENAUER, B.; PARDEY, P. G.; ROSEGRANT, M. W. Ending hunger in our lifetime: food security and globalization. International Food Policy Research Institute, 2003.

SCHULTZ, T. W. Transforming traditional agriculture. Yale University Press, 1964.

THIRTLE, C.; LIN, L.; PIESSE, J. The impact of research-led agricultural productivity growth on poverty reduction in Africa, Asia and Latin America. World Development, v. $31, \quad$ n. 12 , p. 1959-1975, 2003. Disponível em: <http://dx.doi.org/10.1016/j.worlddev.2003.07.001 >. doi: 10.1016/j.worlddev.2003.07.001

TREWAVAS, A. Malthus foiled again and again. Nature, v. 418, n. 6898, p. 668-670, 2002. Disponível em: <http://dx.doi.org/10.1038/nature01013 >. doi: 10.1038/nature01013

VIEIRA FILHO, J. E. R; SILVEIRA, J. M. F. J. da. Mudança tecnológica na agricultura: uma revisão crítica da literatura e o papel das economias de aprendizado. Revista de Economia e Sociologia Rural, v. 50, n. 4, p. 721-742, 2012. Disponível em: <http://dx.doi.org/10.1590/S0103-20032012000400008 >. doi: $10.1590 /$ S0103-20032012000400008

WANG, Y.; ZHOU, Q. Evaluation of development of agricultural modernization in central China. IERI Procedia, v. 4, p. 417-424, 2013. Disponível em: $<$ http://dx.doi.org/10.1016/j.ieri.2013.11.060 >. doi: 10.1016/j.ieri.2013.11.060 
WORLD BANK. Indicators. 2016. Disponível em:< http://data.worldbank.org/indicator\#topic-1 >. Acesso em: 05/06/2016.

ZHANG, F.; ZHAN, J.; ZHANG, Q.; YAN, H.; SUN, Z. Allocating agricultural production factors: a scenario-based modeling of wheat production in Shandong Province, China. Physics and Chemistry of the Earth, Parts A/B/C, 2016. Disponível em: <http://dx.doi.org/10.1016/j.pce.2016.06.002 >. doi: 10.1016/j.pce.2016.06.002

ZHOU, Y.; STAATZ, J. Projected demand and supply for various foods in West Africa: Implications for investments and food policy. Food Policy, v. 61, p. 198-212, 2016. Disponível em: <http://dx.doi.org/10.1016/j.foodpol.2016.04.002 >. doi: 10.1016/j.foodpol.2016.04.002 